\title{
Enfin un gène pour la lipodystrophie congénitale de Berardinelli-Seip
}

La lipodystrophie congénitale de Berardinelli-Seip (BSCL) est un syndrome très rare caractérisé par une quasi-absence de tissu adipeux qui est notée dès la naissance ou au cours des premiers mois de la vie [2-4]. Les autres signes majeurs sont, sur le plan clinique, un acanthosis nigricans*, une hypertrophie musculaire, une hyperandrogénie, une hépatomégalie et, sur le plan biologique, une hypertriglycéridémie, un hyperinsulinisme majeur et souvent un diabète. En outre, des antécédents de diabète sont fréquemment rapportés dans les familles des patients. La fréquence élevée de consanguinité parentale est en faveur d'un mode de transmission autosomique récessif, mais l'origine génétique était jusqu'alors inconnue. Grâce à une collaboration avec Mark Lathrop et son équipe du Centre national de génotypage, des médecins ou chercheurs de différents pays, ainsi que les patients et leur famille, nous venons d'identifier le premier gène dont l'altération est responsable de la BSCL [1].

Les études génétiques antérieures avaient essentiellement recherché l'implication de gènes candidats, c'est-à-dire de gènes dont l'altération pouvait expliquer la résistance à l'insuline ou l'absence de tissu adipeux. Ces études avaient toutes rapporté des résultats négatifs permettant, dans les meilleurs des cas, d'exclure l'implication des gènes étudiés dans la pathogénie de la maladie [5]. Récemment, en utilisant une approche de clonage positionnel, qui vise d'abord à localiser le locus de la maladie puis à identifier le gène, un

* Ensemble de placards hyperkératosiques épaissis, rugueux, hyperpigmentés, siégeant de façon élective dans les grands plis (aisselles, aine, régions latérocervicales), symétriques et non prurigineux et histologiquement caractérisés par une papillomatose avec locus (BSCL1) a été localisé sur le chromosome 9q34 dans plusieurs familles par une équipe nord-américaine mais le gène correspondant n'est toujours pas identifié [6].

Nous avons également utilisé une approche de clonage positionnel sur neuf familles, pour la plupart consanguines, provenant de deux isolats géographiques au Liban et en Norvège. Le criblage systématique du génome des membres de ces familles par une approche de cartographie d'homozygotie - en nous fondant sur le principe que les enfants atteints dans ces isolats géographiques sont homozygotes pour le même allèle hérité d'un ancêtre commun - a révélé un nouveau locus de la maladie, BSCL2. Ce locus est commun aux deux isolats du Liban et de Norvège et est localisé sur le chromosome 11q13 dans l'intervalle flanqué par les marqueurs microsatellites D11S4076 et D11S480 distants approximativement de 2,5 Mb [1]. L'analyse de 20 familles supplémentaires, d'origines ethniques variées, a identifié 11 familles, pour la plupart d'origine portugaise, dans lesquelles la maladie est liée au locus BSCL2. Dans les autres familles qui sont le plus souvent d'origine africaine ou nord-africaine, la maladie n'est pas liée au locus 11q13 mais semble en revanche l'être avec le locus 9q34 précédemment décrit [6].

Le séquençage de 27 gènes situés dans l'intervalle chromosomique 11q13, dans un échantillonnage de 4 patients, nous a permis de détecter une délétion de trois exons d'un de ces gènes chez un patient, ce qui suggérait qu'il puisse correspondre au gène $B S C L 2$ de la maladie [1]. Ce gène est l'homologue humain $d u$ gène murin $G n g 3 l g$, lié à la sous-unité

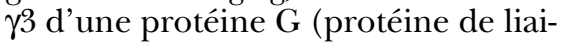
son du GTP) [7]. Gng3lg n'a pas de fonction connue, et son nom est dû à sa proximité sur le chromosome avec le gène codant pour la protéine G- $\gamma 3$. L'analyse de BSCL2 chez 71 patients atteints de BSCL a révélé des mutations chez 44 patients dont tous ceux appartenant aux familles dans lesquelles la maladie est génétiquement liée au locus 11q13. Ces patients sont soit homozygotes (même mutation sur les deux allèles parentaux) soit hétérozygotes composites (une mutation différente sur chaque allèle parental), en accord avec un mode de transmission récessif de la maladie. Au total, 13 mutations différentes ont été identifiées. La plupart sont des mutations "nulles", c'est-àdire qu'elles entraînent la production d'une protéine tronquée probablement inactive [1].

Ainsi, BSCL2 est le premier gène impliqué dans la BSCL. Il code pour une protéine que nous avons appelée "seipine», en l'honneur de Martin Seip qui a décrit la maladie. La seipine est une protéine de 398 acides aminés avec au moins 2 domaines transmembranaires potentiels [1]. Aucune séquence consensus ou motif commun à d'autres protéines connues, n'a été retrouvé et, par conséquent, le rôle physiologique de la seipine reste à déterminer.

Le gène de la seipine est exprimé dans tous les tissus, mais de façon très variable; il est exprimé de façon particulièrement abondante dans toutes les régions neuronales testées du cerveau [1]. Cette expression centrale indique que la maladie pourrait être induite par un signal déclenché dans le cerveau qui a le pouvoir de contrôler le circuit homéostatique et les réponses neuroendocriniennes. Cette observation est compatible avec les rapports cliniques et biologiques originaux qui impliquaient un défaut primaire de l'axe hypothalamo-hypophysaire dans l'étiologie de la BSCL [2-4]. En effet, la présence anormale 
de facteurs de libération hypothalamiques avait été décrite dans le plasma des patients $[8,9]$. Une fraction protéique isolée à partir de l'urine des patients Norvégiens (chez lesquels nous venons de détecter des mutations de la seipine) et injectée chez des animaux avait induit un syndrome anabolique associé à une lipoatrophie et à un diabète, ce phénomène n'apparaissant pas quand le patient donneur ou l'animal receveur étaient hypophysectomisés [10]. Enfin, tous les patients présentent un syndrome anabolique avec une accélération de la croissance initiale et une organomégalie. Ils présentent également une augmentation du métabolisme basal qui est associée à un excès de prise alimentaire malgré une disparition du tissu adipeux.

Ainsi, la découverte de la seipine représente la première étape dans la compréhension de l'étiologie de la BSCL. Il reste encore à déterminer qu'elle est sa fonction physiologique et comment son expression est contrôlée pour établir le mécanisme physiopathologique de la maladie. D'une façon générale, ces études devraient nous apporter des informations nouvelles sur les mécanismes centraux réglant le comportement alimentaire et le métabolisme énergétique ainsi que sur le contrôle et la distribution du tissu adipeux, processus qui sont altérés dans des syndromes plus communs comme le diabète, le syndrome d'insulinorésistance et l'obésité.

1. Magré J, Délépine M, Khallouf E, et al. Identification of the gene altered in Berardinelli-Seip congenital lipodystrophy on chromosome 11q13. Nat Genet 2001; 28 : 365-70.

2. Berardinelli W. An undiagnosed endocrinometabolic syndrome: report of two cases. J Clin Endocrinol Metab 1954; 14: 193-204.

3. Seip M. Lipoatrophy and gigantism with associated endocrine manifestations: a new diencephalic syndrome? Acta Paediatr Scand 1959; 48: $555-74$.

4. Seip M, Trygstad O. Generalized lipodystrophy, congenital and acquired (lipoatrophy). Acta Paediatr Suppl 1996; 413: 2-28.

5. Vigouroux C, Khallouf E, Bourut C, et al. Genetic exclusion of 14 candidate genes in lipoatropic diabetes using linkage analysis in 10 consangui- neous families. J Clin Endocrinol Metab 1997; 82: 3438-44.

6. Garg A, Wilson R, Barnes R, et al. A gene for congenital generalized lipodystrophy maps to human chromosome 9q34. J Clin Endocrinol Metab $1999 ; 84 ; 3390-4$.

7. Downes GB, Copeland NG, Jenkins NA, Gautam N. Structure and mapping of the G protein gamma3 subunit gene and a divergently transcribed novel gene, gng3lg. Genomics 1998; 53 : 22030 .

8. Mabry CC, Hollingsworth DR, Upton GV, Corbin A. Pituitary-hypothalamic dysfunction in generalized lipodystrophy. J Pediatr 1973; 82: 625-33. 9. Corbin A, Upton GV, Mabry CC, Hollingsworth DR. Diencephalic involvement in generalized lipodystrophy: rationale and treatment with the neuroleptic agent, pimozide. Acta Endocrinol (Copenh) 1974; 77 : 209-20.

10. Foss I, Trygstad O. Lipoatrophy produced in mice and rabbits by a fraction prepared from the urine from patients with congenital generalized lipodystrophy. Acta Endocrinol (Copenh) 1975; 80 : 398-416.

\section{Jocelyne Magré}

Inserm U. 402, Laboratoire de biologie cellulaire, Faculté de médecine SaintAntoine, 27, rue Chaligny, 75012 Paris, France.

\section{SOCIÉTÉ DE BIOLOGIE}

\section{Aspects moléculaires des maladies neurodégénératives \\ Coordonnateur: Yves Christen \\ (Fondation IPSEN - Paris) \\ Mercredi 19 décembre 2001, 16 heures}

Yves Christen (Fondation IPSEN - Paris)

Des protéines et des mutations : une nouvelle vision (moléculaire) des maladies neurodégénératives

Alexis Brice (Inserm U. 289 - Paris)

Parkine, $\alpha$-synucléine et autres aspects moléculaires de la maladie de Parkinson

Luc Buée (Inserm U. 422 - Lille)

Tau story: des démences frontales aux autres tauopathies

Frédéric Checler (IPMC du Cnrs-UMR 6097 - Valbonne)

Les maladies dégénératives sont-elles des pathologies du protéasome?

Anne Joutel (Inserm EPI 99-21 - Paris)

Notch, destinées cellulaires et maladies du système nerveux

Institut des Cordeliers, Amphithéâtre Bilski-Pasquier, 15-21, rue de l'École-de-Médecine, 75006 Paris, France Renseignements :

Secrétariat de la Société de Biologie, Collège de France, 3, rue d’Ulm, 75231 Paris Cedex 05, France. Tél./Fax : 0144271340 
